\title{
Wernicke encephalopathy: a medical emergency
}

\author{
Gregory S. Day MD MSc, C. Martin del Campo MD
}

\begin{abstract}
Wernicke encephalopathy is prevalent, yet underdiagnosed
Thiamine (vitamin $\mathrm{B}_{1}$ ) deficiency causing Wernicke encephalopathy occurs most frequently in people with compromised absorption (e.g., malnutrition, hyperemesis), increased metabolism (e.g., sepsis, malignancy) or increased carbohydrate intake (e.g., administration of intravenous dextrose). Guidelines from the European Federation of Neurological Societies suggest that Wernicke encephalopathy is not a rare disorder, but rather a rare diagnosis. ${ }^{1}$ The diagnosis is confirmed in $0.4 \%-2.8 \%$ of autopsies, yet may be overlooked in $68 \%$ of patients with alcoholism and $94 \%$ of patients without alcoholism. ${ }^{1}$
\end{abstract}

\section{Specific criteria have been developed for diagnosis}

Wernicke encephalopathy is a clinical diagnosis. Altered mental state (e.g., confusion, encephalopathy), ocular abnormalities (e.g., nystagmus, ophthalmoplegia) and cerebellar dysfunction (e.g., gait disturbance, ataxia) define this condition. Diagnostic sensitivity can be optimized with the Caine criteria (Box 1). ${ }^{3}$

\section{Box 1: Caine criteria for diagnosis of Wernicke encephalopathy ${ }^{3}$}

A diagnosis of Wernicke encephalopathy should be considered in any patient with two of the following:*

- Nutritional deficiency

- Altered mental state or memory impairment

- Oculomotor abnormalities

- Cerebellar dysfunction

*Sensitivity approaches $100 \%$ in patients with alcoholism without hepatic encephalopathy.
Rapid correction of brain thiamine deficiency is the goal of therapy

Thiamine is an essential vitamin critical to glucose metabolism. Without thiamine, glucose is metabolized through less-efficient anaerobic pathways, which produces lactic acid. Acidosis affecting periventricular structures (i.e., thalami, mammillary bodies, oculomotor nuclei, cerebellar vermis) accounts for the clinical presentation. $^{2}$ Because thiamine crosses the blood-brain barrier via passive and active transport, correction of brain thiamine deficiency can be promoted by maximizing serum thiamine concentrations. ${ }^{4}$ Oral absorption is unreliable in patients at risk of Wernicke encephalopathy, which emphasizes the importance of parenteral treatment. ${ }^{1,4}$

For references, please see Appendix 1, available at www.cmaj.ca/lookup/suppl /doi:10.1503/cmaj.130091/-/DC1

CMAJ invites submissions to "Five things to know about ..." Submit manuscripts online at http://mc.manuscriptcentral.com/cmaj

Wernicke encephalopathy is a medical emergency with substantial morbidity and mortality

A historical case series reported mortality in $17 \%$ of patients with acute Wernicke encephalopathy. ${ }^{2}$ Of the remainder, $84 \%$ given low doses of parenteral thiamine $(50-100 \mathrm{mg} / \mathrm{d})$ had irreversible memory impairment consistent with Korsakoff syndrome; only 16\% made a full recovery.

Some thiamine is good for treatment; more may be better

There is insufficient evidence from randomized controlled trials to dictate optimal treatment for Wernicke encephalopathy caused by alcohol misuse. ${ }^{5}$ Although European guidelines recommend acute treatment with at least $200 \mathrm{mg}$ thiamine intravenously or intramuscularly three times daily for two to three days, ${ }^{1}$ the efficacy of higher doses requires further study. Dramatic resolution of neurologic signs signals adequate treatment and confirms the clinical diagnosis of Wernicke encephalopathy.

Competing interests: C. Martin del Campo has received funds from UCB Canada and Boehringer Ingelheim. No competing interests declared by Gregory Day.

This article has been peer reviewed.

Affiliation: Division of Neurology, University of Toronto; and University Health Network, Toronto, Ont.

Correspondence to: Gregory Day, gregg.day@mail.utoronto.ca

CMAJ 2014. DOI:10.1503/cmaj.130091 